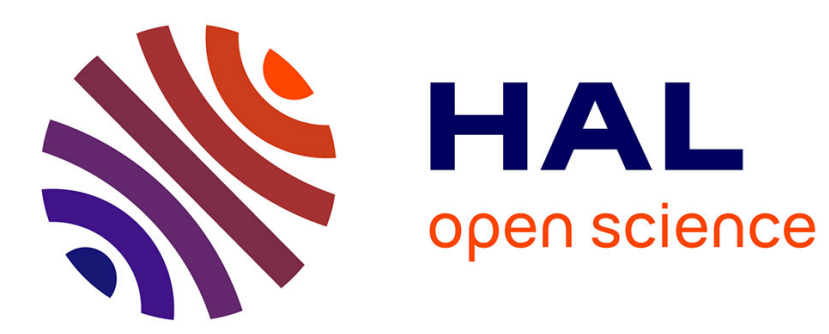

\title{
Local Generic Position for Root Isolation of Zero-dimensional Triangular Polynomial Systems
}

Jia Li, Jinsan Cheng, Elias Tsigaridas

\section{To cite this version:}

Jia Li, Jinsan Cheng, Elias Tsigaridas. Local Generic Position for Root Isolation of Zero-dimensional Triangular Polynomial Systems. CASC 2012 - 14th International Workshop on Computer Algebra in Scientific Computing, Sep 2012, Maribor, Slovenia. pp.186-197, 10.1007/978-3-642-32973-9_16 . hal-00776212

\section{HAL Id: hal-00776212 \\ https://hal.inria.fr/hal-00776212}

Submitted on 15 Jan 2013

HAL is a multi-disciplinary open access archive for the deposit and dissemination of scientific research documents, whether they are published or not. The documents may come from teaching and research institutions in France or abroad, or from public or private research centers.
L'archive ouverte pluridisciplinaire HAL, est destinée au dépôt et à la diffusion de documents scientifiques de niveau recherche, publiés ou non, émanant des établissements d'enseignement et de recherche français ou étrangers, des laboratoires publics ou privés. 


\title{
Local Generic Position for Root Isolation of Zero-dimensional Triangular Polynomial Systems
}

\author{
Jia $\mathrm{Li}^{1}$, Jin-San Cheng ${ }^{2}$, and Elias P. Tsigaridas ${ }^{3}$ \\ ${ }^{1}$ Beijing Electronic Science and Technology Institute lijia@besti.edu.cn \\ 2 KLMM, AMSS, Chinese Academy of Sciences jcheng@amss.ac.cn \\ ${ }^{3}$ POLSYS project, INRIA, LIP6/CNRS elias@polsys.lip6.fr
}

\begin{abstract}
We present an algorithm based on local generic position (LGP) to isolate the complex or real roots and their multiplicities of a zero-dimensional triangular polynomial system. The Boolean complexity of the algorithm for computing the real roots is single exponential: $\tilde{\mathcal{O}}_{B}\left(N^{n^{2}}\right)$, where $N=\max \{d, \tau\}, d$ and $\tau$, is the degree and the maximum coefficient bitsize of the polynomials, respectively, and $n$ is the number of variables.
\end{abstract}

\section{Introduction}

Solving polynomial systems is a basic problem in the fields of computational sciences, engineering, etc. Usually, the polynomial systems are transformed into triangular polynomial systems by algebraic methods, such as Gröbner bases, characteristic sets, $\mathrm{CAD}$, and resultants. In most case, we consider zero-dimensional polynomial systems. So in the end, we need to solve zero-dimensional triangular systems. One practical problem is to determine the topology of real algebraic curves or surfaces with CAD based method $[4,8,17]$, we need to isolate the real roots of a zero-dimensional triangular system with multiple zeros. We will discuss how to solve this kind of system in this paper.

A zero-dimensional triangular system has the form $\Sigma_{n}=\left\{f_{1}, \ldots, f_{n}\right\}$, where $f_{i} \in \mathbb{Q}\left[x_{1}, \ldots, x_{i}\right](i=1, \ldots, n), \mathbb{Q}$ is the field of rational numbers. Our aim is to find zeros $\boldsymbol{\xi}^{n}=\left(\xi_{1}, \ldots, \xi_{n}\right) \in \mathbb{C}^{n}$ ( or $\mathbb{R}^{n}$ ) of $\Sigma_{n}$, where $\mathbb{C}, \mathbb{R}$ are the field of complex and real numbers, respectively.

A local generic position method (shortly LGP) was introduced in [6]. The method was used to solve bivariate polynomial systems and the experiments show that the method works well. The method was extended to solve general zero-dimensional system by computing Gröbner basis at first and then computing a linear univariate representation [7]. In this paper, we will extend the LGP method to solve general zero-dimensional triangular system by computing resultant only. The complexity analysis and the experiments show the effectivities and efficiency of the algorithm.

For the system $\Sigma_{i+1}(i \geq 1)$, we can assume that we have got the zeros of $\Sigma_{i}$. For any fixed zeros of $\Sigma_{i-1}$ (it may be $\left.\{0\}\right),\left(f_{i}, f_{i+1}\right)$ can be regarded as a bivariate polynomial system. We shear the hypersurfaces (surface, curve) 
defined by the polynomials $f_{i}, f_{i+1}$ on a special direction such that the first $i-1$ coordinates and the $(i+1)$-th coordinate are unchanged. The new system is denoted as $\Sigma_{i+1}^{\prime}$. Then we project all the zeros of $\Sigma_{i+1}^{\prime}$ to the $i$-dimensional space by eliminating the $(i+1)$-th coordinate, denoted as $\Sigma_{i}^{*}$. Solving $\Sigma_{i}^{*}$, we can recover the roots of $\Sigma_{i+1}$ by the LGP method with the zeros of $\Sigma_{i}, \Sigma_{i}^{*}$. Step by step, we can get all the zeros of the system. And the method keeps the multiplicity of each zero of the given system. In the end, we get an algebraic representation for the zeros of the system $\Sigma_{n}$ : each coordinate of each zero is a linear combination of some zeros of several univariate polynomials. From the algebraic representation, we can get the zeros of the system under any given precision.

The bit complexity of our algorithm for real roots is $\tilde{\mathcal{O}}_{B}\left(N^{n^{2}}\right)$, where $N, n$ will be defined in Section 4. Our method is complete in the sense that $\Sigma_{n}$ can be any zero-dimensional triangular system.

Root-isolating of zero-dimensional triangular system is studied before. Most of the methods can not deal with triangular system with multiple zeros directly $[10,12,19,5,23]$. Usually, they decompose the system into triangular systems without multiple zeros and then isolate the real zeros of them. Cheng et al [9] provides a direct method, although their method does not give an algebraic representation of the real zeros and can not give the multiplicities of the zeros. In [26], they provide a method to compute the multiplicities of the real zeros when they compute the zero by existing methods. There are some related work about algebraic representation. Gao and Chou [16] privide a method to represent the zeros of a radical characteristic set. From a Gröbner basis, a rational representation of the zeros of a system is provided and the representation depends on the multiplicities of the solutions [1]. Fouillier [21] uses rational univeriate representation to represent the zeros of a polynomial system by computing the Gröbner basis of the system.

\section{Zero-dimensional triangular system solving}

In this section, we give the basic theory for our method.

Let $\Sigma_{i}=\left\{f_{1}\left(x_{1}\right), f_{2}\left(x_{1}, x_{2}\right), \ldots, f_{i}\left(x_{1}, x_{2}, \ldots, x_{i}\right)\right\} \in \mathbb{Q}\left[x_{1}, x_{2}, \ldots, x_{i}\right](i=$ $1, \ldots, n)$ be a general zero-dimensional triangular system. $\boldsymbol{\xi}^{i}=\left(\xi_{1}, \ldots, \xi_{i}\right) \in$ $\operatorname{Zero}\left(\Sigma_{i}\right)$, where Zero $(t)$ represents the zero set of $t=0$. And $t$ can be a polynomial or a polynomial system.

Let $f \in \mathbb{C}[x]$. Then the separation bound $\operatorname{sep}(f)$ and root bound $\operatorname{rb}(f)$ of $f$ are defined as follows: $\operatorname{sep}(f):=\min \{\Delta(\alpha, \beta) \mid \forall \alpha, \beta \in \mathbb{C}$ s.t. $f(\alpha)=f(\beta)=$ $0, \alpha \neq \beta\}$, where $\Delta(\alpha, \beta):=\min \{|\operatorname{Re}(\alpha-\beta)|,|\operatorname{Im}(\alpha-\beta)|\}, \operatorname{Re}(\alpha-\beta), \operatorname{Im}(\alpha-\beta)$ are the real part and imaginary part of $\alpha-\beta$ respectively. We also need the definition of the root bound: $\operatorname{rb}(f):=\max \{|\alpha| \mid \forall \alpha \in \mathbb{C}$ s.t.f $(\alpha)=0\}$.

Assume that we have solved the system $\Sigma_{i}(1 \leq i \leq n-1)$. The assumption is reasonable since we can solve $\Sigma_{1}$ directly with many existing tools, such as $[22,25]$. And we can get a separation bound $r_{1}$ of the roots of $f_{1}\left(x_{1}\right)=0$. Based on the roots of $f_{1}=0$, we can estimate the root bound $R_{2}$. 
Let $r_{j}(1 \leq j \leq i)$ be a positive rational number, such that

$$
r_{j} \leq \frac{1}{2} \min _{\boldsymbol{\xi}^{j-1} \in \operatorname{Zero}\left(\Sigma_{j-1}\right)} \operatorname{sep}\left(f_{j}\left(\boldsymbol{\xi}^{j-1}, x_{j}\right)\right) .
$$

We can compute $r_{j}$ after we get the roots of $f_{j}\left(\boldsymbol{\xi}^{j-1}, x_{j}\right)=0$.

Based on the zeros of $\Sigma_{j}$, we can estimate the root bound on $x_{j+1}$ (we will show how to estimate the bound later) to get a positive rational number $R_{j+1}$, such that

$$
R_{j+1} \geq \max _{\boldsymbol{\xi}^{j} \in \operatorname{Zero}\left(\Sigma_{j}\right)} \operatorname{rb}\left(f_{j+1}\left(\boldsymbol{\xi}^{j}, x_{j+1}\right)\right) .
$$

We usually add a previously estimated value, say $r_{j+1}^{\prime}$, for $r_{j+1}$ to the above root bound to ensure that after shearing and projection, the fixed neighborhoods of the zeros of $T_{i}^{i}\left(X_{i}^{i}\right)$ (see definition below) are disjoint. Then when we compute $r_{j+1}$, we choose the one no larger than $r_{j+1}^{\prime}$.

We say two plane curves defined by $f, g \in \mathbb{C}[x, y]$ s.t. $\operatorname{gcd}(f, g)=1$ are in a generic position w.r.t. $y$ if (1) The leading coefficients of $f$ and $g$ w.r.t. $y$ have no common factors, and (2) If $h$ is the resultant of $f$ and $g$ w.r.t. $y$, then any $\alpha \in \mathbb{C}$ such that $h(\alpha)=0, f(\alpha, y), g(\alpha, y)$ have only one common zero in $\mathbb{C}$.

Now we introduce local generic position $[6,7]$. Given $f, g \in \mathbb{Q}[x, y]$, not necessarily in generic position, we considerthe the mapping $\phi:(x, y) \rightarrow(x+s y, y), s \in$ $\mathbb{Q}$, with the following properties: (i) $\phi(f), \phi(g)$ are in a generic position w.r.t. $y$, and (ii) Let $\bar{h}, h$ be the resultants of $\phi(f), \phi(g)$ and $f, g$ w.r.t. $y$, respectively. Each root $\alpha$ of $h(x)=0$ has a neighbor interval $H_{\alpha}$ such that $H_{\alpha} \cap H_{\beta}=\emptyset$ for roots $\beta \neq \alpha$ of $h=0$. And any root $(\gamma, \eta)$ of $f=g=0$ which has a same $x$-coordinate $\gamma$, is mapped to $\gamma^{\prime}=\gamma+s \eta \in H_{\gamma}$, where $h(\gamma)=0, \bar{h}\left(\gamma^{\prime}\right)=0$, as shown in Figure 1. Thus we can recover $\eta=\frac{\gamma^{\prime}-\gamma}{s}$.

\subsection{Basic theory and method}

For each $\boldsymbol{\xi}^{i}=\left(\xi_{1}, \ldots, \xi_{i}\right) \in \operatorname{Zero}\left(\Sigma_{i}\right)$, the roots of $f_{i+1}\left(\boldsymbol{\xi}^{i}\right.$, $\left.x_{i+1}\right)=0$ are bounded by $R_{i+1}$. We can take a shear mapping on $f_{i+1}\left(x_{1}, \ldots, x_{i+1}\right)$ such that when projected to $i$-D space, all the roots of $f_{i+1}\left(\boldsymbol{\xi}^{i}, x_{i+1}\right)=0$ are projected into the fixed neighborhood of $\xi_{i}$ (centered at $\xi_{i}$ bounded by $r_{i} / 2$ ). This can be achieved by take the following shear mapping on $\left(x_{i}, x_{i+1}\right)$.

$$
X_{2}^{i+1}=x_{i}+\frac{r_{i}}{R_{i+1}} x_{i+1}, X_{1}^{i+1}=x_{i+1} .
$$

Applying (3) to the system $\Sigma_{i+1}$, we derive a new system $\Sigma_{i+1}^{\prime}=\left\{f_{1}\left(x_{1}\right), \ldots\right.$, $f_{i-1}\left(x_{1}, \ldots, x_{i-1}\right), f_{i}\left(x_{1}, \ldots, x_{i-1}, X_{2}-\frac{r_{i}}{R_{i+1}} X_{1}^{i+1}\right)$,

$\left.f_{i+1}\left(x_{1}, \ldots, x_{i-1}, X_{2}^{i+1}-\frac{r_{i}}{R_{i+1}} X_{1}^{i+1}, X_{1}^{i+1}\right)\right\}$. There is only one root of $f_{i+1}\left(\xi_{1}, \ldots, \xi_{i-1}, \theta_{2}-\right.$ $\left.\frac{r_{i}}{R_{i+1}} X_{1}^{i+1}, X_{1}^{i+1}\right)=0$ corresponding to each $i$-D root $\left(\xi_{1}, \ldots, \xi_{i-1}, \theta_{2}\right) \in \operatorname{Zero}\left(\Sigma_{i}^{*}\right)$.

As is shown in Figure 1, $\theta_{2}$ is some dot point on $x_{i}$-axis, corresponding to each dot point, there is only one triangle point. Let

$$
\begin{gathered}
T_{2}^{i+1}\left(x_{1}, \ldots, x_{i-1}, X_{2}^{i+1}\right)=\operatorname{Res}_{X_{1}^{i+1}}\left(f _ { i } \left(x_{1}, \ldots, x_{i-1}, X_{2}^{i+1}-\right.\right. \\
\left.\left.\frac{r_{i}}{R_{i+1}} X_{1}^{i+1}\right), f_{i+1}\left(x_{1}, \ldots, x_{i-1}, X_{2}^{i+1}-\frac{r_{i}}{R_{i+1}} X_{1}^{i+1}, X_{1}^{i+1}\right)\right),
\end{gathered}
$$




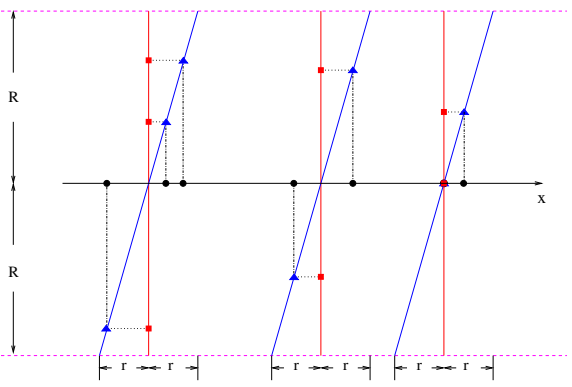

Fig. 1. Local generic position

where $\operatorname{Res}_{t}(f, g)$ is the resultant of $f$ and $g$ w.r.t. $t$. Then we get a triangular system $\Sigma_{i-1} \cap\left\{T_{2}^{i+1}\right\}$. We will further study the relationship between the zeros of $\Sigma_{i+1}$ and $\Sigma_{i-1} \cap\left\{T_{2}^{i+1}\right\}$ below. Considering the multiplicities of the zeros, we give the following lemma.

Lemma 1. For each zero $\boldsymbol{\xi}^{i}$ of $\Sigma_{i-1}$, there exists a one to one correspondence between the roots of $\left\{f_{i}\left(\xi_{1}, \ldots, \xi_{i-1}, x_{i}\right), f_{i+1}\left(\xi_{1}, \ldots, \xi_{i-1}, x_{i}, x_{i+1}\right)\right\}=0$ and the roots of $T_{2}^{i+1}\left(\xi_{1}, \ldots, \xi_{i-1}, X_{2}^{i+1}\right)=0$, and the multiplicities of corresponding zeros in their equation(s) are the same.

Lemma 2. There exists a one to one correspondence between the zeros of triangular systems $\Sigma_{i+1}$ and $\Sigma_{i-1} \cap\left\{T_{2}^{i+1}\left(x_{1}, \ldots, x_{i-1}, X_{2}^{i+1}\right)\right\}$. And the corresponding zeros have the same multiplicities in their system.

Proof. Since both the systems have a same sub-system $\Sigma_{i-1}$, we can derive that the lemma is correct by Lemma 1 .

Lemma 3. For $\left(\xi_{1}, \ldots, \xi_{i}\right) \in \operatorname{Zero}\left(\Sigma_{i}\right)$, the roots of $f_{i+1}\left(\xi_{1}, \ldots, \xi_{i}, x_{i+1}\right)$ are:

$$
x_{i+1}=\frac{R_{i+1}}{r_{i}}\left(\zeta_{2}-\xi_{i}\right), T_{2}^{i+1}\left(\xi_{1}, \ldots, \xi_{i-1}, \zeta_{2}\right)=0 \text { and }\left|\zeta_{2}-\xi_{i}\right|<r_{i} .
$$

Proof. The first formula is directly derived from (3). Note that the first formula just holds for $\zeta_{2}$ ' corresponding zeros having $\xi_{i}$ as coordinate. So the inequality holds.

The above lemma tells us how to derive the roots of $f_{i+1}\left(\xi_{1}, \ldots, \xi_{i}, x_{i+1}\right)=0$ from the roots of $T_{2}^{i+1}\left(\xi_{1}, \ldots, \xi_{i-1}, X_{2}^{i+1}\right)=0$. From (1) and (2), the corollary below is obvious.

Corollary 1. All the roots of $T_{2}^{i+1}\left(\xi_{1}, \ldots, \xi_{i-1}, X_{2}^{i+1}\right)=0$ are inside the fixed neighborhood of 0 (centered at 0 bounded by $\left.R_{i}\right)$ for all $\left(\xi_{1}, \ldots, \xi_{i-1}\right) \in \operatorname{Zero}\left(\Sigma_{i-1}\right)$.

We apply the previous procedure on the triangular system $\Sigma_{i-1} \cap\left\{T_{2}^{i+1}\right\}$ with the mapping

$$
X_{3}^{i+1}=x_{i-1}+\frac{r_{i-1}}{R_{i}} X_{2}^{i+1}, X_{2}^{i+1}=X_{2}^{i+1},
$$


we can derive

$$
\begin{gathered}
T_{3}^{i+1}\left(x_{1}, \ldots, x_{i-2}, X_{3}^{i+1}\right)=\operatorname{Res}_{X_{2}^{i+1}}\left(f _ { i - 2 } \left(x_{1}, \ldots, x_{i-3}, X_{3}^{i+1}-\right.\right. \\
\left.\left.\frac{r_{i-1}}{R_{i}} X_{2}^{i+1}\right), T_{2}^{i+1}\left(x_{1}, \ldots, x_{i-3}, X_{3}^{i+1}-\frac{r_{i-1}}{R_{i}} X_{2}^{i+1}, X_{2}^{i+1}\right)\right) .
\end{gathered}
$$

So, we have a triangular system $\Sigma_{i-2} \cap\left\{T_{3}^{i+1}\right\}$. Since Corollary 1 holds, the results in Lemma 2 still hold on $\Sigma_{i-1} \cap\left\{T_{2}^{i+1}\right\}$ and $\Sigma_{i-2} \cap\left\{T_{3}^{i+1}\right\}$. By (5), and similarly as (4), we derive

$$
\zeta_{2}=\frac{R_{i}}{r_{i-1}}\left(\zeta_{3}-\xi_{i-1}\right),\left|\zeta_{3}-\xi_{i-1}\right|<r_{i-1}, \quad T_{3}^{i+1}\left(\xi_{1}, \ldots, \xi_{i-2}, \zeta_{3}\right)=0 .
$$

Then we have $x_{i+1}=\frac{R_{i+1}}{r_{i}}\left(\frac{R_{i}}{r_{i-1}}\left(\zeta_{3}-\xi_{i-1}\right)-\xi_{i}\right)$, where $\left|\zeta_{3}-\xi_{i-1}\right|<r_{i-1}$, $\left|\frac{R_{i}}{r_{i-1}}\left(\zeta_{3}-\xi_{i-1}\right)-\xi_{i}\right|<r_{i}, T_{3}^{i+1}\left(\xi_{1}, \ldots, \xi_{i-2}, \zeta_{3}\right)=0$.

The above formula means that we can get the roots of $f_{i+1}\left(\xi_{1}, \ldots, \xi_{i}, x_{i+1}\right)=$ 0 by solving $T_{3}^{i+1}\left(\xi_{1}, \ldots, \xi_{i-2}, X_{3}^{i+1}\right)=0$ directly.

Step by step, we can derive a univariate polynomial $T_{i+1}^{i+1}\left(X_{i+1}^{i+1}\right)$. It holds $\zeta_{i}=$ $\frac{R_{2}}{r_{1}}\left(\zeta_{i+1}-\xi_{1}\right)$ and $\left|\zeta_{i+1}-\xi_{1}\right|<r_{1}$. Now we can represent Zero $\left(f_{i+1}\left(\xi_{1}, \ldots, \xi_{i}, x_{i+1}\right)\right)$ by $\xi_{1}, \ldots, \xi_{i}$ and the roots of $T_{i+1}^{i+1}\left(X_{i+1}^{i+1}\right)$, where $\left(\xi_{1}, \ldots, \xi_{i}\right) \in \operatorname{Zero}\left(\Sigma_{i}\right)$.

Lemma 4. For any zero $\left(\xi_{1}, \ldots, \xi_{i}\right) \in \operatorname{Zero}\left(\Sigma_{i}\right)$, each root $\xi_{i+1}$ of $f_{i+1}\left(\xi_{1}, \ldots, \xi_{i}, x_{i+1}\right)=$ 0 is mapped to a root of $T_{i+1}^{i+1}\left(X_{i+1}^{i+1}\right)=0$. And we can derive $\xi_{i+1}$ by $T_{i+1}^{i+1}\left(X_{i+1}^{i+1}\right)=$ 0 as follows.

$$
\begin{gathered}
\xi_{i+1}=\frac{R_{i+1}}{r_{i}}\left(\zeta_{2}-\xi_{i}\right), \quad \zeta_{2}=\frac{R_{i}}{r_{i-1}}\left(\zeta_{3}-\xi_{i-1}\right), \\
\ldots, \\
\zeta_{i}=\frac{R_{2}}{r_{1}}\left(\zeta_{i+1}-\xi_{1}\right), T_{i+1}^{i+1}\left(X_{i+1}^{i+1}\right)=0,
\end{gathered}
$$

where $\left|\zeta_{2}-\xi_{i}\right|<r_{i},\left|\zeta_{3}-\xi_{i-1}\right|<r_{i-1}, \ldots,\left|\zeta_{i+1}-\xi_{1}\right|<r_{1}$.

Proof. Using Lemma 3 recursively, we can derive the above formula.

Lemma 5. For any $\left(\xi_{1}, \ldots, \xi_{i}\right) \in \operatorname{Zero}\left(\Sigma_{i}\right)$, each distinct root $\xi_{i+1}$ of $f_{i+1}\left(\xi_{1}, \ldots, \xi_{i}, x_{i+1}\right)=$ 0 is mapped to a root of $T_{i+1}^{i+1}\left(X_{i+1}^{i+1}\right)=0$. And we can derive $\xi_{i+1}$ as follows.

$$
\xi_{i+1}=\left(\prod_{j=1}^{i} \frac{R_{j+1}}{r_{j}}\right)\left(\eta_{i+1}-\eta_{i}\right),
$$

where $\eta_{i+1} \in \operatorname{Zero}\left(T_{i+1}^{i+1}\right), \eta_{i} \in \operatorname{Zero}\left(T_{i}^{i}\right)$, and $\left|\eta_{i+1}-\eta_{i}\right|<\left(\prod_{j=1}^{i-1} \frac{r_{j}}{R_{j+1}}\right) r_{i}$.

Lemma 6. The multiplicity of the zero $\left(\xi_{1}, \ldots, \xi_{i}, \xi_{i+1}\right)$ of $\Sigma_{i+1}$ is equal to the multiplicity of the corresponding root in $T_{i+1}^{i+1}\left(X_{i+1}^{i+1}\right)=0$.

Proof. Using Lemma 2 recursively, we can derive the lemma. 
Theorem 1. Wih the notations above, we have the following representation for a general zero-dimensional triangular system $\Sigma_{n}:\left\{\left\{T_{1}^{1}, \ldots, T_{n}^{n}\right\},\left\{r_{1}, \ldots, r_{n-1}\right\}\right.$, $\left.\left\{R_{2}, \ldots, R_{n}\right\}\right\}$, such that the zeros of $\Sigma_{n}$ can be derived as follows.

$$
\begin{aligned}
\xi_{1}= & \eta_{1}, \eta_{1} \in \operatorname{Zero}\left(T_{1}^{1}\right), \\
\xi_{2}= & \frac{R_{2}}{r_{1}}\left(\eta_{2}-\eta_{1}\right), \eta_{2} \in \operatorname{Zero}\left(T_{2}^{2}\right),\left|\eta_{2}-\eta_{1}\right|<r_{1}, \\
& \cdots \\
\xi_{i}= & \left(\prod_{j=1}^{i-1} \frac{R_{j+1}}{r_{j}}\right)\left(\eta_{i}-\eta_{i-1}\right), \eta_{i} \in \operatorname{Zero}\left(T_{i}^{i}\right), \\
& \quad\left|\eta_{i+1}-\eta_{i}\right|<\left(\prod_{j=1}^{i-1} \frac{r_{j}}{R_{j+1}}\right) r_{i}, \\
& \ldots \\
\xi_{n}= & \left(\prod_{j=1}^{n-1} \frac{R_{j+1}}{r_{j}}\right)\left(\eta_{n}-\eta_{n-1}\right), \eta_{n} \in \operatorname{Zero}\left(T_{n}^{n}\right), \\
& \quad\left|\eta_{n}-\eta_{n-1}\right|<\left(\prod_{j=1}^{n-2} \frac{r_{j}}{R_{j+1}}\right) r_{n-1},
\end{aligned}
$$

where $T_{j}^{j}(j=1, \ldots, n)$ are univariate polynomials, $T_{1}^{1}=f_{1}$. For each zero $\left(\xi_{1}, \ldots, \xi_{i}\right)(1 \leq i \leq n)$ of the system $\Sigma_{i}$, the multiplicity of the zero in the system is the multiplicity of the corresponding zero $\eta_{i}$ in the univariate polynomial $T_{i}^{i}$.

Remark: From the second part of the theorem, we can compute the multiplicity of $\xi_{i+1} \in \operatorname{Zero}\left(f_{i+1}\left(\xi_{1}, \ldots, \xi_{i}, x_{i+1}\right)\right)$ in $f_{i+1}\left(\xi_{1}, \ldots, \xi_{i}, x_{i+1}\right)=0$, it is the multiplicity of the zero $\left(\xi_{1}, \ldots, \xi_{i+1}\right)$ in $\Sigma_{i+1}$ dividing the multiplicity of the zero $\left(\xi_{1}, \ldots, \xi_{i}\right)$ in $\Sigma_{i}$. It gives a simple proof for the main result in [23].

\subsection{Estimation of bounds $r_{i}, R_{i+1}$}

To estimate the bounds $r_{i}, R_{i+1}$, we can directly derive the bound by the method in [14]. But the derived bounds $r_{i}$ is tiny and $R_{i+1}$ is huge. We prefer to use direct methods to get the bounds.

For $r_{i}$, we can directly compute the bound on the zeros of $\Sigma_{i}$ using (1). Let

$$
S\left(x_{i+1}\right)=\operatorname{Res}_{x_{1}}\left(\operatorname{Res}_{x_{2}}\left(\cdots \operatorname{Res}_{x_{i}}\left(f_{i+1}, f_{i}\right), \cdots, f_{2}\right), f_{1}\right) .
$$

Then we can estimate $R_{i+1}$ by estimating the root bound of $S\left(x_{i+1}\right)$.

The methods to estimate the bound for $r_{i}, R_{i+1}$ can be used both for complex and real roots isolation. We focus on real roots isolation in this paper. So for $r_{i}$, we compute it after we get the real roots of $\Sigma_{i}=0$ with the following formula. $\operatorname{sep}(f):=\min \{|\alpha-\beta| \mid \forall \alpha, \beta \in \mathbb{R}$ s.t. $f(\alpha)=f(\beta)=0, \alpha \neq \beta\}$.

For $R_{i+1}$, we at first estimate the root bound on $f_{i+1}\left(\xi_{1}, \ldots, \xi_{i}, x_{i+1}\right)=0$ for a fixed zero $\left(\xi_{1}, \ldots, \xi_{i}\right)$. Doing so, we need to use the definition of sleeve (see $[9,18,19]$ for details). Given $g \in \mathbb{Q}\left[x_{1}, \ldots, x_{n}\right]$, we decompose it uniquely as $g=$ $g^{+}-g^{-}$, where $g^{+}, g^{-} \in \mathbb{Q}\left[x_{1}, \ldots, x_{n}\right]$ each has only positive coefficients and with minimal number of monomials. Given an isolating box $\square \boldsymbol{\xi}^{i}=\left[a_{1}, b_{1}\right] \times \cdots \times\left[a_{i}, b_{i}\right]$ for $\boldsymbol{\xi}^{i}=\left(\xi_{1}, \ldots, \xi_{i}\right)$, we assume that $a_{j}, b_{j}, \xi_{j} \geq 0,1 \leq j \leq i$ since we can take a coordinate system transformation to satisfy the condition when $\xi_{j}<0$. Then we define

$$
f^{u}(x)=f_{i+1}^{u}\left(\square \boldsymbol{\xi}^{i} ; x\right)=f_{i+1}^{+}\left(\boldsymbol{b}_{i}, x\right)-f_{i+1}^{-}\left(\boldsymbol{a}_{i}, x\right),
$$




$$
f^{d}(x)=f_{i+1}^{d}\left(\square \boldsymbol{\xi}^{i} ; x\right)=f_{i+1}^{+}\left(\boldsymbol{a}_{i}, x\right)-f_{i+1}^{-}\left(\boldsymbol{b}_{i}, x\right),
$$

where $\boldsymbol{a}_{i}=\left(a_{1}, \ldots, a_{i}\right), \boldsymbol{b}_{i}=\left(b_{1}, \ldots, b_{i}\right)$. Then $\left(f^{u}, f^{d}\right)$ is a sleeve of $f_{i+1}\left(\boldsymbol{\xi}^{i}, x_{i+1}\right)$. When considering $x \geq 0$, we have (see [9]):

$$
f^{d}(x) \leq f_{i+1}\left(\boldsymbol{\xi}^{i}, x\right) \leq f^{u}(x) .
$$

If the leading coefficients of $f_{u}$ and $f_{d}$ have the same signs, then we can find that the root bound of $f_{i+1}\left(\boldsymbol{\xi}^{i}, x\right)$ is bounded by the root bounds of $f_{u}$ and $f_{d}$.

Lemma 7. [24] Let a polynomial of degree d be $f(x)=a_{d} x^{d}+a_{d-1} x^{d-1}+\ldots+$ $a_{0} \in \mathbb{R}[x], a_{d} \neq 0$. Let $R=1+\max _{0 \leq k \leq d-1}\left|\frac{a_{k}}{a_{d}}\right|$, then all zeros of $f(x)$ lie inside the circle of radius $R$ about the origin.

If the considered triangular system is not regular, the leading coefficients of $f_{u}$ and $f_{d}$ always have different signs. But the absolute value of the leading coefficients are very close to zero. So usually, the root bound of $f_{i+1}\left(\boldsymbol{\xi}^{i}, x\right)$ is also bounded by the larger of the root bound of $f_{u}$ and $f_{d}$. Then we can get $R_{i+1}$ by the lemma above.

The ways to compute $r_{i}, R_{i+1}$ for real case usually work for our method since a random shear mapping usually puts the system into a generic position and the real roots are in a local generic position.

\subsection{Precision control}

When we compute the approximating zeros of a given zero-dimensional triangular system with the method we provided, the errors of the zeros will cumulate. So we need to control the error under a wanted precision. This is what we want to discuss in this subsection.

Consider the coordinate $\xi_{i}$ of the zero $\boldsymbol{\xi}^{\boldsymbol{n}}=\left(\xi_{1}, \ldots, \xi_{n}\right)$ of the triangular system $\Sigma_{n}$ in Theorem 1. Assume that we derive the coordinate $\xi_{j}$ under the precision $\rho_{j}(>0)$, and we isolate the roots of $T_{j}^{j}\left(X_{j}^{j}\right)=0$ under the precision $\epsilon_{j}(>0)$, Note that $\rho_{1}=\epsilon_{1}$.

From (8), the following lemma is clear.

Lemma 8. With the symbol above, we can derive that the root precision $\rho_{i}$ for $\xi_{i}$ is defined as follows.

$$
\rho_{i}=\left(\prod_{j=1}^{i-1} \frac{R_{j+1}}{r_{j}}\right)\left(\epsilon_{i}+\epsilon_{i-1}\right) .
$$

From Lemma 8, we can compute the zeros of $\Sigma_{n}$ under any given precision by controlling the precisions $\epsilon_{i}(1 \leq i \leq n)$. For example, we can set them as follows if we require the precision of the output zeros to be $\epsilon$.

$$
\epsilon_{i}=\Pi_{j=1}^{i} \frac{r_{j}}{R_{j+1}} \frac{\epsilon}{2}(1 \leq i \leq n-1), \epsilon_{n}=\Pi_{j=1}^{n-1} \frac{r_{j}}{R_{j+1}} \frac{\epsilon}{2} .
$$

In order to practically avoid refining the roots when we want to control the precision under a given $\epsilon$, we can previously assume $\frac{R_{i+1}}{r_{i}}$ less than a number, 
such as $10,2^{3}$, before we solve the system. This help us to previously estimate the precisions that should be used to get the roots of $T_{i}^{i}\left(X_{i}^{i}\right)=0(1 \leq i \leq n)$.

For root isolation, we require not only the roots satisfying the given precision, but the isolating boxes being disjoint for distinct roots. We will show how to ensure that the isolating boxes are disjoint.

For real numbers $\alpha$ and $\beta, \alpha<\beta$ in $\mathbb{R}$, if we use intervals $[a, b]$ and $[c, d]$ to represent them respectively. Denote

$$
|\alpha|=|b-a|, \operatorname{Dis}(\alpha, \beta)= \begin{cases}c-b, & b<c \\ 0, & b \geq c .\end{cases}
$$

For real points $\xi=\left(\xi_{1}, \ldots, \xi_{n}\right)$ and $\eta=\left(\eta_{1}, \ldots, \eta_{n}\right)$ in $\mathbb{R}^{n}$, if we use boxes $\left[a_{1}, b_{1}\right] \times \ldots \times\left[a_{n}, b_{n}\right]$ and $\left[c_{1}, d_{1}\right] \times \ldots \times\left[c_{n}, d_{n}\right]$ to represent them respectively. Denote

$$
|\xi|=\max _{i=1, \ldots, n}\left\{b_{i}-a_{i}\right\}, \operatorname{Dis}(\xi, \eta)=\min _{i=1, \ldots, n}\left\{\operatorname{Dis}\left(\xi_{i}, \eta_{i}\right)\right\} .
$$

If $\operatorname{Dis}(\xi, \eta)>0$, we say $\xi$ and $\eta$ are disjoint.

Theorem 2. With the notations above. We use intervals to represent real numbers and use boxes to represent real points in the computation, if for any $\eta_{i}^{j} \in$ $\operatorname{Zero}\left(T_{i}^{i}\right), \eta_{i-1} \in \operatorname{Zero}\left(T_{i-1}^{i-1}\right),\left|\eta_{i}^{j}-\eta_{i-1}\right|<\left(\prod_{j=1}^{i-2} \frac{r_{j}}{R_{j+1}}\right) r_{i}, i=2, \ldots, n ; j=1,2$,

$$
\operatorname{Dis}\left(\eta_{i}^{1}, \eta_{i}^{2}\right)>\left|\eta_{i-1}\right|
$$

then any two real zeros $\boldsymbol{\xi}^{1}=\left(\xi_{1}^{1}, \ldots, \xi_{n}^{1}\right)$ and $\boldsymbol{\xi}^{2}=\left(\xi_{1}^{2}, \ldots, \xi_{n}^{2}\right)$ of $\Sigma_{n}$ are disjoint.

\section{The main algorithm}

Algorithm 3 Isolate the real (or complex) roots of a 0-dim. triangular system.

Input: $A$ zero-dimensional triangular system $\Sigma_{n}$, a precision $\epsilon$.

Output: The solutions of the system in isolating interval representation.

1. Isolate the real (or complex) roots of $f_{1}\left(x_{1}\right)=0$ under the precision $\rho=\frac{\epsilon}{20}$.

Let $T_{1}^{1}\left(X_{1}^{1}\right)=f_{1}\left(X_{1}^{1}\right)$.

2. For $i$ from 2 to $n$,

(a) Estimate $r_{i-1}$ with method in Section 2.2.

(b) Estimate $R_{i}$ with method in Section 2.2.

(c) Compute $T_{i}^{i}\left(X_{i}^{i}\right)$ with method in Section 2.1.

(d) Isolate the real roots of $T_{i}^{i}\left(X_{i}^{i}\right)=0$ with precision $\prod_{j=1}^{i-1} \frac{r_{j}}{R_{j+1}} \frac{\epsilon}{20}\left(\Pi_{j=1}^{n-1} \frac{r_{j}}{R_{j+1}} \frac{\epsilon}{2}\right.$ if $i=n)$. Compute the multiplicities of the real roots if needed when $i=n$.

(e) If (14) is not satisfied, then refine the real (or complex) roots of $T_{i-1}^{i-1}\left(X_{i-1}^{i-1}\right)=$ 0 until (14) is satisfied.

(f) Recover the real zeros of $\Sigma_{i}$ from $T_{i}^{i}\left(X_{i}^{i}\right)$ and $\Sigma_{i-1}$ by Theorem 1.

3. Get the algebraic solutions of $\Sigma_{n}:\left\{\left\{T_{1}^{1}\left(X_{1}^{1}\right), \ldots\right.\right.$,

$\left.\left.T_{n}^{n}\left(X_{n}^{n}\right)\right\},\left\{r_{1}, \ldots, r_{n-1}\right\},\left\{R_{2}, \ldots, R_{n}\right\}\right\}$

Or numeric solutions and their corresponding multiplicities. 
Example 4 Consider the system $\left\{x^{2}-6,5 x^{2}+10 x y+6 y^{2}-5, x^{2}+2 x y+\right.$ $\left.2 y^{2}+4 y z+5 z^{2}-1\right\}$. We derive a symbolic representation of the roots, as well as a floating point approximation up to precision $\frac{1}{10^{3}}$. We isolate the roots of $f_{1}=0$ using precision $\frac{1}{2 \cdot 10^{4}}$ and we derive the zero set:

$$
H=\left\{\xi_{1}^{1}=-2.449490070, \xi_{1}^{2}=2.449490070\right\} .
$$

Let $r_{1}=2$. Consider $\xi_{1} \approx-2.449490070 \in[-2.45,-2.44]$. We can use $-2.45,-2.44$ to construct $f^{u}(y), f^{d}(y)$ for $f_{2}\left(\xi_{1}, y\right)$. We compute a root bound for $f^{u}(y), f^{d}(y)$. For both it is $\leq 6$. Similarly, we compute a root bound for the other root in $H$. we notice that all the root bounds are less than 6 . We have computed $r_{2}=2$, so we set $R_{2}=6+2=2^{3}$. By considering a coordinate system transformation, we derive a system $\Sigma_{2}^{\prime}$ as follows

$$
\left\{X_{2}^{22}-\frac{1}{2} X_{2}^{2} X_{1}^{2}+\frac{1}{16} X_{1}^{2^{2}}-6,5 X_{2}^{22}+\frac{15}{2} X_{2}^{2} X_{1}^{2}+\frac{61}{16} X_{1}^{2^{2}}-5\right\}
$$

Hence we can compute $T_{2}^{2}=36 X_{2}^{2^{4}}-\frac{1083}{4} X_{2}^{2^{2}}+\frac{130321}{256}$. Solve $T_{2}^{2}\left(X_{2}^{2}\right)=0$ under the precision $\frac{1}{8 \cdot 10^{4}}$, we have its real roots and multiplicities (the number in each bracket is the multiplicity of the root in the system): $G=\left\{\eta_{2}^{1}=\right.$ $\left.-1.939178944[2], \eta_{2}^{2}=1.939178944[2]\right\}$.

For each root $\eta_{2}$ in $G$, if it satisfies $\left|\eta_{2}-\xi_{1}\right|<r_{1}=2$, then it corresponds to $\xi_{1}$, where $\xi_{1}$ is a root in $H$. And the multiplicity of $\left(\xi_{1}, \eta_{2}\right)$ in the given system is the corresponding multiplicity of $\eta_{2}$ in $T_{2}^{2}=0$. In this way, we can get the approximating roots of the subsystem $\Sigma_{2}$ :

$$
\{[-2.449490070 \text { [1], } 2.041244504 \text { [2]], [2.449490070 [1], -2.041244504 [2]]] }
$$

With the method of Section 2.2, we estimate $r_{3}=2$, and we derive that 3 is a bound for the $z$ coordinate. Let $R_{3}=2+2=4$ and $r_{2}=2$ and consider a coordinate system transformation as mentioned above. By computing the resultant, we can get

$$
T_{3}^{3}=810000 x^{8}-13500000 x^{6}+84375000 x^{4}-234375000 x^{2}+244140625
$$

Then, we get the solution of the given triangular system as follows.

$$
\begin{aligned}
& \left\{\left\{X_{1}^{1^{2}}-6,36 X_{2}^{2^{4}}-\frac{1083}{4} X_{2}^{2^{2}}+\frac{130321}{256},\right.\right. \\
& \left.\left.810000 x^{8}-13500000 x^{6}+84375000 x^{4}-234375000 x^{2}+244140625\right\},\{2,2\},\{8,4\}\right\}
\end{aligned}
$$

We solve $T_{3}^{3}$ using precision $\frac{1}{16 \cdot 10^{4}}$, and derive its roots and multiplicities:

$$
J=\left\{\eta_{3}^{1}=-2.041241452[4], \eta_{3}^{2}=2.041241452[4]\right\} .
$$

For each root $\eta_{3}$ in $J$, if it satisfies $\left|\eta_{3}-\eta_{2}\right|<\frac{r_{1}}{R_{2}} r_{2}=\frac{1}{2}$, then it corresponds to the same $\left(\xi_{1}, \xi_{2}\right)$ with $\eta_{2}$, where $\left(\xi_{1}, \xi_{2}\right)$ is a root in $\Sigma_{2}$. And the multiplicity of $\left(\xi_{1}, \xi_{2}, \eta_{3}\right)$ in the given system is the corresponding multiplicity of $\eta_{3}$. In this way, we can get the approximating roots of the system:

$$
\begin{aligned}
& \{[-2.449490070[1], 2.041244504[2],-0.816497800[4]], \\
& [2.449490070[1],-2.041244504[2], 0.816497800[4]]\}
\end{aligned}
$$

Using Lemma 8, the precision of roots is $4\left(\frac{1}{8 \cdot 10^{4}}+\frac{1}{16 \cdot 10^{4}}\right)<\frac{1}{10^{3}}$. 


\section{Complexity Analysis}

In what follows $\mathcal{O}_{B}$ means bit complexity and the $\tilde{\mathcal{O}}_{B}$-notation means that we are ignoring logarithmic factors. For a polynomial $f \in \mathbb{Z}[X], \operatorname{deg}(f)$ denotes its degree. By $\mathcal{L}(f)$ we denote an upper bound on the bit size of the coefficients of $f$ (including a bit for the sign), $\tilde{\mathcal{O}}$ indicates that we omit logarithmic factors. For $a \in \mathbb{Q}, \mathcal{L}(a)$ is the maximum bit size of the numerator and the denominator.

Lemma 9. [22]For a polynomial $f$ of degree $d$ with integer coefficients of modulus less than $2^{\tau}$, we can isolate the real roots of $f$ in $\tilde{\mathcal{O}}_{B}\left(d^{3} \tau\right)$.

Lemma 10. [2, 24] Let $f(x)$ be a polynomial in $\mathbb{Z}[x]$ and $\operatorname{deg}_{x}(f) \leq d, \mathcal{L}(f) \leq$ $\tau$. Then the separation bound of $f$ is $\operatorname{sep}(f) \geq d^{-\frac{d+2}{2}}(d+1)^{\frac{1-d}{2}} 2^{\tau(1-d)}$, thus $\log (\operatorname{sep}(f))=\tilde{\mathcal{O}}(d \tau)$. The latter provides a bound on the bit size of the endpoints of the isolating intervals.

Lemma 11. [11]Let $f, g \in\left(Z\left[y_{1}, \ldots, y_{k}\right]\right)[x]$ with $\operatorname{deg}_{x}(f)=p \geq q=\operatorname{deg}_{x}(g)$, $\operatorname{deg}_{y_{i}}(f) \leq p$ and $\operatorname{deg}_{y_{i}}(g) \leq q, \mathcal{L}(f)=\tau \geq \sigma=\mathcal{L}(g)$. We can compute $\operatorname{Res}(f, g)$ w.r.t. $x$ in $\tilde{\mathcal{O}}_{B}\left(q(p+q)^{k+1} p^{k} \tau\right)$. And $\operatorname{deg}_{y_{i}}\left(\operatorname{Res}_{x}(f, g)\right) \leq 2 p q$, and the bit size of resultant is $\tilde{\mathcal{O}}(p \sigma+q \tau)$.

Lemma 12. Let $\Sigma_{k+1}=\left\{f_{1}\left(x_{1}\right), f_{2}\left(x_{1}, x_{2}\right), \ldots, f_{k+1}\left(x_{1}, x_{2}, \ldots, x_{k+1}\right)\right\} \in \mathbb{Z}\left[x_{1}, \ldots, x_{k+1}\right]$. Assume $\operatorname{deg}_{x_{j}}\left(f_{i}\left(x_{1}, x_{2}, \ldots, x_{i}\right)\right) \leq d, \mathcal{L}\left(f_{i}\left(x_{1}, \ldots, x_{i}\right)\right) \leq \tau, 1 \leq j \leq i, 1 \leq i \leq$ $k+1$. For any real numbers $\left\{\xi_{1}, \ldots, \xi_{k}\right\} \in \operatorname{Zero}\left(\Sigma_{k}\right)$ represented by intervals $\left[a_{1}, b_{1}\right], \ldots,\left[a_{k}, b_{k}\right]$, assume $\mathcal{L}\left(\xi_{i}\right) \leq \sigma_{i}$. Then we compute $R_{k+1}$ in $\tilde{\mathcal{O}}_{B}\left(k d^{\frac{(k+2)^{2}}{2}}\right)$ and $\mathcal{L}\left(R_{k+1}\right) \leq \max \left\{\tilde{\mathcal{O}}\left(\sum_{i=1}^{k} \sigma_{i} d+\tau\right), \tilde{\mathcal{O}}\left(d^{k} \tau\right)\right\}$

Let $\mathbf{x}_{i}$ be the list $x_{1}, \ldots, x_{i}$. For $1 \leq i \leq n, 1 \leq l \leq i-1$, let

$$
\begin{aligned}
\varphi_{l}^{i}: \mathbb{Z}\left[\mathbf{x}_{i-l+1}\right] & \rightarrow \mathbb{Q}\left[\mathbf{x}_{i-l-1}, X_{l+1}^{i}, X_{l}^{i}\right] \\
f\left(\mathbf{x}_{i-l+1}\right) & \mapsto f\left(\mathbf{x}_{i-l-1}, X_{l+1}^{i}-\frac{r_{l}}{R_{l+1}} X_{l}^{i}, X_{l}^{i}\right)
\end{aligned}
$$

Theorem 5. Let $\Sigma_{n}=\left\{f_{1}\left(x_{1}\right), f_{2}\left(x_{1}, x_{2}\right), \ldots, f_{n}\left(x_{1}, x_{2}, \ldots, x_{n}\right)\right\} \in \mathbb{Z}\left[x_{1}, x_{2}, \ldots, x_{n}\right]$. Assume $\operatorname{deg}_{x_{j}}\left(f_{i}\left(x_{1}, x_{2}, \ldots, x_{i}\right)\right) \leq d, \mathcal{L}\left(f_{i}\left(x_{1}, x_{2}, \ldots, x_{i}\right)\right) \leq \tau$, where $1 \leq j \leq i$, and $1 \leq i \leq n$. Using Alg. 3 to isolate the real roots of $\Sigma_{n}$, we deduce:

$-\mathcal{L}\left(\xi_{i}\right)=\tilde{\mathcal{O}}\left(d^{i^{2}+2 i-2} \tau\right), 1 \leq i \leq n$,

$-\mathcal{L}\left(r_{i}\right)=\tilde{\mathcal{O}}\left(d^{i^{2}+2 i-2} \tau\right)$, and $\mathcal{L}\left(R_{i+1}\right)=\tilde{\mathcal{O}}\left(d^{i^{2}+2 i-1} \tau\right)$, where $1 \leq i \leq n-1$,

$-\operatorname{deg}_{X_{l}^{i}} T_{l}^{i}\left(\mathbf{x}_{i-l}, X_{l}^{i}\right) \leq 3^{l-1} d^{l}, \operatorname{deg}_{x_{j}} T_{l}^{i}\left(\mathbf{x}_{i-l}, X_{l}^{i}\right) \leq 2^{l-1} d^{l}, \mathcal{L}\left(T_{l}^{i}\right)=\tilde{\mathcal{O}}\left(d^{i^{2}+l-2} \tau\right)$ where $2 \leq i-l, 2 \leq l \leq i, 2 \leq i \leq n$,

- We compute $T_{l}^{i}$ in $\tilde{\mathcal{O}}_{B}\left(d^{i^{2}+(2 l-2) i-2 l^{2}+6 l-5} \tau\right)$, and $\left\{\xi_{i}\right\}$ in $\tilde{\mathcal{O}}_{B}\left(d^{i^{2}+4 i-2} \tau\right)$, where $2 \leq l \leq i, 2 \leq i \leq n$.

Theorem 6. The complexity of Alg. 3 is $\tilde{\mathcal{O}}_{B}\left(N^{n^{2}}\right)$, where $N=\max \{d, \tau\}$. 


\section{Experiments}

In this section, we illustrate the function of our algorithm by some examples. The timings are collected on a computer running Maple 15 with $2.29 \mathrm{GHz} \mathrm{CPU}$, $2 \mathrm{G}$ memory and Windows XP by using the time command in Maple.

We compare our method with Discover, Isolate, EVB and Vincent-CollinsAkritas algorithm. Discoverer is a tool for solving problems about polynomial equations and inequalities [23]. Isolate is a tool to solve general equation systems based on Realsolving C library by Rouillier. EVB is developed by Cheng et al in [9]. Vincent-Collins-Akritas algorithm which isolates real roots for univariate polynomials uses techniques which are very close to the ones used by Rioboo in [20]. Sqf is the method in [9] for zero-dimensional triangular system without multiple roots. All the required precision are 0.001 .

In Table 1, we compare different methods by computing some zero-dimensional triangular polynomial systems without multiple roots. All the tested systems have the form $\left(f_{1}, f_{2}, \ldots, f_{n}\right)$. And $\operatorname{deg}\left(f_{i}\right)=k$ are the degrees of the polynomials. We take average timings for different degrees (each degree with several random examples).

In Table 2, we take polynomials with three variables. They are surfaces, denoted as $f$, in $\mathbb{R}^{3}$. We compute the resultant of $f$ and $\frac{\partial f}{\partial z}$ with respect to $z$. Denote its squarefree part as $g$. Then we compute the resultant of $g$ and $\frac{\partial g}{\partial y}$ with respect to $y$ and denote the squarefree part as $h$. Thus we get a triangular polynomial system $\{h, g, f\}$. When computing the topology of real algebraic surfaces, one usually needs to solve this kind of triangular system. It is usually zero-dimensional. This kind of system always have multiple roots. We test this kind of zero-dimensional triangular systems for the methods which can deal with multiple roots directly. They are Isolate, EVB and LGP.

From the data, we can find that LGP works well for system with multiple roots comparing to the existing direct method. For the systems without multiple roots, Sqf is the most efficient method. LGP works well for system with fewer roots. For the systems with higher degrees or more variables, that is, systems with more roots, LGP will slow down comparing to other methods. The reason is that $\prod_{j=1}^{i-1} \frac{r_{j}}{R_{j+1}}$ becomes small, thus the resultant computations take much more time.

Acknowledgment. Partially supported by the EXACTA grant of the National Science Foundation of China (NSFC 60911130369) and the French National Re- search Agency (ANR-09-BLAN-0371-01).

\section{References}

1. M.-E. Alonso, E. Becker, M.-F. Roy, and T. Wörmann. Multiplicities and idempotents for zero dimensional systems. In Algorithms in algebraic Geometry and Applications, Vol. 143 of Progress in Mathematics, pages 1-20. Birkhäuser, 1996.

2. S. Basu, R. Pollack, and M. F. Roy. Algorithms in Real Algebraic Geometry, Volume 10 of Algorithms and Computation in Mathematics, Springer-Verlag, 2003.

3. E. Becker and T. Wörmann. Radical computations of zero-dimensional ideals and real root counting. Math. and Computers in Simulation, 42(4-6):561-569, 1996. 
4. E. Berberich, M. Kerber, and M. Sagraloff. Exact Geometric-Topological Analysis of Algebraic Surfaces. In M. Teillaud, editor, Proc. of the 24th ACM Symp. on Computational Geometry (SoCG), pages 164-173. ACM press, 2008.

5. F. Boulier, C. Chen, F.Lemaire and M. Moreno Maza. Real Root Isolation of Regular Chains. ASCM 2009: 1-15, 2009.

6. J. S. Cheng, X. S. Gao, and J. Li. Root isolation for bivariate polynomial systems with local generic position method. ISSAC 2009: 103-110, 2009.

7. J. S. Cheng, X. S. Gao, and L. Guo. Root isolation of zero-dimensional polynomial systems with linear univariate representation. J. of Symbolic Computation (2011).

8. J. S. Cheng, X. S. Gao, and M. Li. Determining the topology of real algebraic surfaces. In Mathematics of Surfaces XI, pp. 121-146, LNCS3604, Springer, 2005.

9. J. S. Cheng, X. S. Gao, and C. K. Yap. Complete Numerical Isolation of Real Roots in 0-dimensional Triangular Systems, JSC, 44(7): 768-785 (2009).

10. G. E. Collins, J. R. Johnson, and W. Krandick. Interval arithmetic in cylindrical algebraic decomposition. Journal of Symbolic Computation, 34:145-157, 2002.

11. D. I. Diochnos, I. Z. Emiris, and E. P. Tsigaridas. On the asymptotic and practical complexity of solving bivariate systems over the reals, J. of Symbolic Computation, 44(7):818-835,2009.

12. A. Eigenwillig, L. Kettner, W. Krandick, K. Mehlhorn, S. Schmitt, and N. Wolpert. A Descartes algorithm for polynomials with bit stream coefficients. in CASC 2005: 138-49, LNCS 3718, Springer, 2005.

13. I. Z. Emiris, B. Mourrain, and E. P. Tsigaridas. Real Algebraic Numbers: Complexity Analysis and Experimentation, LNCS 5045, pages 57-82, 2008.

14. I. Z. Emiris, B. Mourrain, and E. P. Tsigaridas. The DMM bound: Multivariate (aggregate) separation bounds. ISSAC 2010: 243-250, ACM, Germany, July 2010.

15. W. Fulton. Introduction to intersection theory in algebraic geometry, Volume 54 of CBMS Regional Conference Series in Mathematics. Published for the Conference Board of the Mathematical Sciences, Washington, DC, 1984

16. X. S. Gao and S. C. Chou. On the theory of resolvents and its applications, Mathematics and Systems Science, 1997.

17. H. Hong. An Efficient Method for Analyzing the Topology of Plane Real Algebraic Curves. Mathematics and Computers in Simulation, 42:571-582, 1996.

18. H. Hong and V. Stahl. Safe start region by fixed points and tightening. Computing, 53(3-4):323-335, 1994.

19. Z. Lu, B. He, Y. Luo and L. Pan. An Algorithm of Real Root Isolation for Polynomial Systems. SNC 2005.

20. R. Rioboo, Computation of the real closure of an ordered field, ISSAC 1992, Academic Press, San Francisco.

21. F. Rouillier. Solving zero-dimensional systems through the rational univariate representation. AAECC, 9:433-461, 1999.

22. M. Sagraloff. When Newton meets Descartes: A Simple and Fast Algorithm to Isolate the Real Roots of a Polynomial,CoRR abs/1109.6279: (2011)

23. B. Xia and T. Zhang. Real Solution Isolation Using Interval Arithmetic, Computers and Mathematics with Applications, 52:853-860, 2006.

24. C. Yap. Fundamental Problems of Algorithmic Algebra, Oxford University Press, New York, 2000.

25. C. Yap, M. Sagraloff. A simple but exact and efficient algorithm for complex root isolation. ISSAC 2011: 353-360.

26. Z.H. Zhang, T. Fang and B.C. Xia, Real solution isolation with multiplicity of 0-dimensional triangular systems, Science China: Information Sciences, 54(1): 6069, 2011. 


\section{A Proofs}

Table 1. Timing of Real Root Isolation of System without Multiple Roots (Seconds)

\begin{tabular}{|c|c||r|r|r|r|r|r|}
\hline Degree & Vars & LGP & Dis & Iso & VCA & Sleeve & Sqf \\
\hline $2-11$ & 2 & 0.155 & 0.325 & 0.254 & 1.071 & 0.887 & 0.024 \\
$12-20$ & 2 & 4.224 & 3.242 & 23.106 & 7.915 & 39.438 & 0.076 \\
$2-4$ & 3 & 0.113 & 0.336 & 0.202 & 1.774 & 0.152 & 0.045 \\
$5-7$ & 3 & 9.063 & 3.118 & 45.771 & 11.178 & 79.953 & 0.110 \\
$2-3$ & 4 & 0.175 & 0.498 & 0.715 & 2.115 & 0.199 & 0.024 \\
4 & 4 & 10.008 & 2.727 & 70.350 & 13.250 & 24.041 & 0.121 \\
\hline
\end{tabular}

Table 2. Timing of Real Root Isolate of surfaces(Seconds)

\begin{tabular}{|c||r|r|r|}
\hline Degree & LGP & Iso & EVB \\
\hline 2 & 0.205 & 0.225 & 0.092 \\
3 & 1.288 & 16.681 & 3.589 \\
4 & 16.180 & 200.594 & 2337.999 \\
\hline
\end{tabular}

Proof (of Lemma 1). Note that we derive the system

$\Theta_{2}:=\left\{f_{i}\left(\xi_{1}, \ldots, \xi_{i-1}, X_{2}^{i+1}-\frac{r_{i}}{R_{i+1}} X_{1}^{i+1}\right), f_{i+1}\left(\xi_{1}, \ldots, \xi_{i-1}, X_{2}^{i+1}-\frac{r_{i}}{R_{i+1}} X_{1}^{i+1}, X_{1}^{i+1}\right)\right\}$ from the system

$\Theta_{1}:=\left\{f_{i}\left(\xi_{1}, \ldots, \xi_{i-1}, x_{i}\right), f_{i+1}\left(\xi_{1}, \ldots, \xi_{i-1}, x_{i}, x_{i+1}\right)\right\}$

by coordinate system transformation. So there exists a one to one correspondence between their zeros, including the multiplicities of the zeros by the properties of LGP method. And the coordinate system transformation ensures that for any zero $\left(\xi_{i}, \xi_{i+1}\right)$, when projected to $x_{i}$-axis by LGP method, the zero is in the fixed neighborhood of $\xi_{i}$ (centered at $\xi_{i}$ bounded by $r_{i} / 2$ ). This ensures that all the zeros of $\Theta_{2}$, when projected to $x_{i}$-axis, do not overlap, which means any root of $T_{2}^{i+1}\left(\xi_{1}, \ldots, \xi_{i-1}, X_{2}^{i+1}\right)=0$ corresponds to one zero of $\Theta_{2}$. So there exists a one to one correspondence between roots of $T_{2}^{i+1}\left(\xi_{1}, \ldots, \xi_{i-1}, X_{2}^{i+1}\right)=0$ and the zeros of $\Theta_{1}$. It is not difficult to find that the degree of the polynomial $f_{i}\left(\xi_{1}, \ldots, \xi_{i-1}, X_{2}^{i+1}-\frac{r_{i}}{R_{i+1}} X_{1}^{i+1}\right)$ w.r.t. $X_{1}^{i+1}$ is equal to its total degree. And $T_{2}^{i+1}\left(\xi_{1}, \ldots, \xi_{i-1}, X_{2}^{i+1}\right)$ is the resultant of the two polynomials in $\Theta_{2}$ w.r.t. $X_{1}^{i+1}$. Based on the theory in Section 1.6 in [15], we can conclude that the multiplicities of the roots in $T_{2}^{i+1}\left(\xi_{1}, \ldots, \xi_{i-1}, X_{2}^{i+1}\right)=0$ equals the multiplicities of the corresponding zeros of $\Theta_{2}$, and then $\Theta_{1}$. So we derive that the lemma is true. 
Proof (of Lemma5). According to Lemma 4, we know

$$
\begin{aligned}
\xi_{i}= & \frac{R_{i}}{r_{i-1}}\left(\zeta_{2}-\xi_{i-1}\right) \\
= & \frac{R_{i}}{r_{i-1}}\left(\frac{R_{i-1}}{r_{i-2}}\left(\zeta_{3}-\xi_{i-2}\right)-\xi_{i-1}\right) \\
= & \left(\prod_{j=1}^{i-1} \frac{R_{j+1}}{r_{j}}\right) \eta_{i}-\sum_{k=1}^{i-1}\left[\left(\prod_{j=k}^{i-1} \frac{R_{j+1}}{r_{j}}\right) \xi_{k}\right] .
\end{aligned}
$$

Note that here $\zeta_{i}=\eta_{i}$. Similarly, we have

$$
\begin{aligned}
\xi_{i+1}= & \left(\prod_{j=1}^{i} \frac{R_{j+1}}{r_{j}}\right) \eta_{i+1}-\sum_{k=1}^{i}\left[\left(\prod_{j=k}^{i} \frac{R_{j+1}}{r_{j}}\right) \xi_{k}\right] \\
= & \left(\prod_{j=1}^{i} \frac{R_{j+1}}{r_{j}}\right) \eta_{i+1}-\sum_{k=1}^{i-1}\left[\left(\prod_{j=k}^{i} \frac{R_{j+1}}{r_{j}}\right) \xi_{k}\right] \\
& -\frac{R_{i+1}}{r_{i}} \xi_{i} \\
= & \left(\prod_{j=1}^{i} \frac{R_{j+1}}{r_{j}}\right) \eta_{i+1}-\frac{R_{i+1}}{r_{i}} \sum_{k=1}^{i-1}\left[\left(\prod_{j=k}^{i-1} \frac{R_{j+1}}{r_{j}}\right) \xi_{k}\right] \\
& -\frac{R_{i+1}}{r_{i}} \xi_{i} \\
= & \left(\prod_{j=1}^{i} \frac{R_{j+1}}{r_{j}}\right) \eta_{i+1}-\frac{R_{i+1}}{r_{i}} \sum_{k=1}^{i-1}\left[\left(\prod_{j=k}^{i-1} \frac{R_{j+1}}{r_{j}}\right) \xi_{k}\right] \\
& -\frac{R_{i+1}}{r_{i}}\left(\left(\prod_{j=1}^{i-1} \frac{R_{j+1}}{r_{j}}\right) \eta_{i}-\sum_{k=1}^{i-1}\left[\left(\prod_{j=k}^{i-1} \frac{R_{j+1}}{r_{j}}\right) \xi_{k}\right]\right) \\
= & \left(\prod_{j=1}^{i} \frac{R_{j+1}}{r_{j}}\right)\left(\eta_{i+1}-\eta_{i}\right) .
\end{aligned}
$$

Then we have

$$
\begin{aligned}
\left|\eta_{i+1}-\eta_{i}\right| & =\prod_{j=1}^{i} \frac{r_{j}}{R_{j+1}}\left|\xi_{i+1}\right| \\
& <\prod_{j=1}^{i} \frac{r_{j}}{R_{j+1}} R_{i+1} \\
& =\left(\prod_{j=1}^{i-1} \frac{r_{j}}{R_{j+1}}\right) r_{i} .
\end{aligned}
$$

The lemma has been proved.

Proof (of Thm. 2). We need only to consider the case $\eta_{i}^{1}, \eta_{i}^{2}$ are in the neighborhood of $\eta_{i-1}$. Otherwise, they are obviously disjoint. According to (8), for any $i=2, \ldots, n$,

$$
\begin{aligned}
\xi_{i}^{1} & =\left(\prod_{j=1}^{i-1} \frac{R_{j+1}}{r_{j}}\right)\left(\eta_{i}^{1}-\eta_{i-1}\right), \\
\xi_{i}^{2} & =\left(\prod_{j=1}^{i-1} \frac{R_{j+1}}{r_{j}}\right)\left(\eta_{i}^{2}-\eta_{i-1}\right) .
\end{aligned}
$$

If (14) is satisfied,

$$
\begin{aligned}
\operatorname{Dis}\left(\xi_{i}^{1}, \xi_{i}^{2}\right) & =\left(\prod_{j=1}^{i-1} \frac{R_{j+1}}{r_{j}}\right) \operatorname{Dis}\left(\eta_{i}^{1}-\eta_{i-1}, \eta_{i}^{2}-\eta_{i-1}\right) \\
& \geq\left(\prod_{j=1}^{i-1} \frac{R_{j+1}}{r_{j}}\right)\left(\operatorname{Dis}\left(\eta_{i}^{1}, \eta_{i}^{2}\right)-\left|\eta_{i-1}\right|\right) \\
& >0
\end{aligned}
$$

So, $\operatorname{Dis}\left(\boldsymbol{\xi}^{1}, \boldsymbol{\xi}^{2}\right)>0$.

Proof (of Lemma 12). According to section 2.2, we may get $R_{k+1}$ using two different methods in two different cases.

In the first case, we compute $R_{k+1}$ by $(2) . R_{k+1}$ is the maximal one in the root bounds of $f_{k+1}\left(\xi_{1}, \ldots, \xi_{k}, x_{k+1}\right)$ for all $\left\{\xi_{1}, \ldots, \xi_{k}\right\} \in \operatorname{Zero}\left(\Sigma_{k}\right)$. The root bound of $f_{k+1}\left(\xi_{1}, \ldots, \xi_{k}, x_{k+1}\right)$ is the larger between the root bound of 
$f_{k+1}^{u}\left(x_{k+1}\right)$ and $f_{k+1}^{d}\left(x_{k+1}\right)$. Note that $f_{k+1}^{u}\left(x_{k+1}\right)=f_{k+1}^{+}\left(b_{1}, \ldots, b_{k}, x_{k+1}\right)-$ $f_{k+1}^{-}\left(a_{1}, \ldots, a_{k}, x_{k+1}\right) \in \mathbb{Q}\left[x_{k+1}\right]$ is a polynomial with degree less than $d$ and bit size bounded by $\sum_{i=1}^{k} \sigma_{i} d+\tau$. By lemma 7 , if $\mathcal{L}(f) \leq \tau$, then $\mathcal{L}(R) \leq \tau$ where $R$ is the root bound of $f(x)$. Then the bit size of the root bound of $f_{k+1}^{u}(x)$ is bounded by $\sum_{i=1}^{k} \sigma_{i} d+\tau$. Similarly, the bit size of the root bound of $f_{k+1}^{d}\left(x_{k+1}\right)$ is bounded by $\sum_{i=1}^{k} \sigma_{i} d+\tau$.

In the second case, we compute $R_{k+1}$ by computing the root bound of $S\left(x_{k+1}\right)$ defined in $(9)$. First we prove that we can compute $S\left(x_{k+1}\right)$ in $\tilde{\mathcal{O}}_{B}\left(k d^{\frac{(k+2)^{2}}{2}}\right)$ and $\mathcal{L}\left(S\left(x_{k+1}\right)\right) \leq \tilde{\mathcal{O}}\left(d^{k} \tau\right)$. Define

$$
\begin{aligned}
S_{2} & =\operatorname{Res}_{x_{k}}\left(f_{k+1}, f_{k}\right), \\
& \cdots, \\
S_{i+1} & =\operatorname{Res}_{x_{k-i+1}}\left(S_{i}, f_{k-i+1}\right), \\
& \cdots, \\
S_{k+1} & =\operatorname{Res}_{x_{1}}\left(S_{k}, f_{1}\right) .
\end{aligned}
$$

Then $S=S_{k+1}$. We prove following conclusions by inductive method:

(1) We compute $S_{i+1}=\operatorname{Res}_{x_{k-i+1}}\left(S_{i}, f_{k-i+1}\right)$ in

$$
\tilde{\mathcal{O}}_{B}\left(d^{2 i(k-i+2)} \tau\right) \leq \tilde{\mathcal{O}}_{B}\left(d^{\frac{(k+2)^{2}}{2}} \tau\right)
$$

$(2) \mathcal{L}\left(S_{i}\right) \leq \tilde{\mathcal{O}}\left(d^{i} \tau\right)$.

For $i=1$, by lemma 11 , we compute $S_{2}$ in $\tilde{\mathcal{O}}_{B}\left(d(2 d)^{k+1} d^{k} \tau\right)=\tilde{\mathcal{O}}_{B}\left(d^{2(k+1)} \tau\right) \leq$ $\tilde{\mathcal{O}}_{B}\left(d^{\frac{(k+2)^{2}}{2}} \tau\right)$ and $\mathcal{L}\left(S_{2}\right) \leq \tilde{\mathcal{O}}(2 d \tau)=\tilde{\mathcal{O}}(d \tau), \operatorname{deg}_{x_{j}}\left(S_{2}\right) \leq 2 d^{2}$.

Assume we prove above conclusions for $1,2, \ldots, i-1$.

For $i, S_{i+1}=\operatorname{Res}_{x_{k-i+1}}\left(S_{i}, f_{k-i+1}\right) . \mathcal{L}\left(S_{i}\right) \leq 2^{i-1} d^{i-1} \tau$ and $\operatorname{deg}_{x_{j}}\left(S_{i}\right) \leq$ $2^{i-1} d^{i}$. According to lemma 11 , we compute $S_{i+1}$ in $\tilde{\mathcal{O}}_{B}\left(d\left(2^{i-1} d^{i}+d\right)^{k-i+2}\left(2^{i-1} d^{i}\right)^{k-i+1} 2^{i-1} d^{i-1} \tau\right)=$ $\tilde{\mathcal{O}}_{B}\left(d^{2 i(k-i+2)} \tau\right) \leq \tilde{\mathcal{O}}_{B}\left(d^{\frac{(k+2)^{2}}{2}} \tau\right.$ and $\mathcal{L}\left(S_{i+1}\right) \leq \tilde{\mathcal{O}}\left(2^{i-1} d^{i-1} \tau d+2^{i-1} d^{i} \tau\right)=$ $\tilde{\mathcal{O}}\left(d^{i} \tau\right)$.

Hence, we have proved above two conclusions.

According to above discussion, we can compute $S\left(x_{k+1}\right)$ in

$$
\tilde{\mathcal{O}}_{B}\left(k d^{\frac{(k+2)^{2}}{2}} \tau\right)
$$

and $\mathcal{L}\left(S_{k+1}\right) \leq \tilde{\mathcal{O}}\left(d^{k} \tau\right)$. By lemma 7 , the bit size of $\operatorname{rb}\left(S\left(x_{k+1}\right)\right)$ is bounded by $\tilde{\mathcal{O}}\left(d^{k} \tau\right)$.

In conclusion, we proved this lemma.

Proof (of Thm. 5). For any $k=1,2, \ldots, n$, let $\mathcal{L}\left(r_{k}\right), \mathcal{L}\left(R_{k+1}\right), \mathcal{L}\left(\xi_{k}\right)$ be bounded by $\rho_{k}, \tau_{k+1}, \sigma_{k}$ respectively. Furthermore, we can always assume $\xi_{k}$ to be represented by an interval $\left[a_{k}, b_{k}\right]$ where $a_{k}, b_{k}$ are fractions with denominators in the form of $2^{t_{k}}\left(t_{k} \leq \sigma_{k}\right)$ and numerator being $1, r_{k}$ to be in the form $\frac{1}{2^{p_{k}}}\left(p_{k} \leq \rho_{k}\right)$ and $R_{k+1}$ to be in the form $2^{q_{k+1}}\left(q_{k+1} \leq \tau_{k+1}\right)$. Then rational number $\frac{r_{k}}{R_{k+1}}$ are in the form $\frac{1}{2^{p_{k}+q_{k+1}}}$ $\left(p_{k}+q_{k+1} \leq \rho_{k}+\tau_{k+1}\right)$. 
We prove this theorem using inductive method.

For $i=1$, we will compute $\left\{\xi_{1}\right\}, r_{1}, R_{2}$. According to Lemma 9 and Lemma 10 , we isolate $\left\{\xi_{1}\right\}$ in $\tilde{\mathcal{O}}_{B}\left(d^{3} \tau\right)$, and $\mathcal{L}\left(b_{1}\right) \leq \tilde{\mathcal{O}}(d \tau), \mathcal{L}\left(r_{1}\right) \leq \tilde{\mathcal{O}}(d \tau)$. According to Lemma $12, \mathcal{L}\left(R_{2}\right) \leq \max \left\{\tilde{\mathcal{O}}\left(d^{2} \tau+\tau\right), \tilde{\mathcal{O}}_{B}(d \tau)\right\}=\tilde{\mathcal{O}}\left(d^{2} \tau\right)$. Then $(\mathrm{a})(\mathrm{b})(\mathrm{c})(\mathrm{f})$ is correct for $i=1$.

For $i=2$, we will compute $T_{2}^{2},\left\{\xi_{2}\right\}, r_{2}, R_{3}$.

$T_{2}^{2}\left(X_{2}^{2}\right)=\operatorname{Res}_{X_{1}^{2}}\left(\left(2^{\tau_{2}+\rho_{1}}\right)^{d} \varphi_{1}^{2}\left(f_{1}\right),\left(2^{\tau_{2}+\rho_{1}}\right)^{d} \varphi_{1}^{2}\left(f_{2}\right)\right)$, where $\left(2^{\tau_{2}+\rho_{1}}\right)^{d} \varphi_{1}^{2}\left(f_{1}\right)$ and $\left.\left(2^{\tau_{2}+\rho_{1}}\right)^{d} \varphi_{1}^{2}\left(f_{2}\right)\right)$ are polynomials with integer coefficients. Furthermore, $\left(2^{\tau_{2}+\rho_{1}}\right)^{d} \varphi_{1}^{2}\left(f_{1}\right)$ is degree less than $d$ w.r.p.t $X_{1}^{2}$ and bit size less than $\tilde{\mathcal{O}}\left(2\left(\tau_{2}+\right.\right.$ $\left.\left.\rho_{1}\right) d+\tau\right)=\tilde{\mathcal{O}}\left(d \tau_{2}\right)=\tilde{\mathcal{O}}\left(d^{3} \tau\right)$. Similarly, $\left.\left(2^{\tau_{2}+\rho_{1}}\right)^{d} \varphi_{1}^{2}\left(f_{2}\right)\right) \in \mathbb{Z}\left[X_{1}^{2}\right.$,

$\left.X_{2}^{2}\right]$ is degree less than $2 d$ w.r.t. $X_{1}^{2}$ and bit size less than $\tilde{\mathcal{O}}\left(d^{3} \tau\right)$. According to Lemma 11, we compute $T_{2}^{2}\left(X_{2}^{2}\right)$ in $\tilde{\mathcal{O}}_{B}\left(d(2 d+d)^{1+1}(2 d) d^{3} \tau\right)=\tilde{\mathcal{O}}_{B}\left(18 d^{7} \tau\right)$, and $\operatorname{deg}_{X_{2}^{2}}\left(T_{2}^{2}\right) \leq 3 d^{2}$, bit size of $T_{2}^{2}$ is bounded by $\tilde{\mathcal{O}}\left(3 d^{4} \tau\right), T_{2}^{2} \in \mathbb{Z}\left[X_{2}^{2}\right]$. By Lemma 9 , we isolate $\left\{\eta_{2}\right\}$, the real roots of $T_{2}^{2}$ in $\tilde{\mathcal{O}}_{B}\left(3^{4} d^{10} \tau\right)=\tilde{\mathcal{O}}_{B}\left(d^{10} \tau\right)$, and the end points of the isolate intervals of them have bit size bounded by $\mathcal{\mathcal { O }}\left(3^{2} d^{6} \tau\right)=$ $\tilde{\mathcal{O}}\left(d^{6} \tau\right)$. Then, we get back $\left\{\xi_{2}\right\}$ by $\xi_{2}=\frac{R_{2}}{r_{1}}\left(\eta_{2}-\xi_{1}\right)$, the bit size of $\xi_{2}$ are bounded by $\tilde{\mathcal{O}}\left(\tau_{2}+\rho_{1}+\mathcal{L}\left(\eta_{2}\right)\right)=\tilde{\mathcal{O}}\left(\left(d^{6}+d^{3}+d\right) \tau\right)=\tilde{\mathcal{O}}\left(d^{6} \tau\right)$. So $\mathcal{L}\left(r_{2}\right) \leq \tilde{\mathcal{O}}\left(d^{6} \tau\right)$. According to Lemma $12, \mathcal{L}\left(R_{3}\right) \leq \max \left\{\tilde{\mathcal{O}}\left(d^{7} \tau+d^{2} \tau+\tau\right), \tilde{\mathcal{O}}_{B}\left(d^{2} \tau\right)\right\}=\tilde{\mathcal{O}}\left(d^{7} \tau\right)$. Obviously, (a)-(f) have been proved for $i=2$. Assume conclusions have been proved for $1,2, \ldots, i-1$.

For $i$, we will compute $T_{l}^{i}, l=2,3, \ldots, i,\left\{\xi_{i}\right\}, r_{i}, R_{i+1}$.

We will induce $l$ in the following discussion.

For $l=2$, we will compute $T_{2}^{i} . T_{2}^{i}\left(X_{2}^{i}\right)=\operatorname{Res}_{X_{1}^{i}}\left(\left(2^{\tau_{i}+\rho_{i-1}}\right)^{d}\right.$

$\left.\varphi_{i-1}^{i}\left(f_{i-1}\right),\left(2^{\tau_{i}+\rho_{i-1}}\right)^{d} \varphi_{i-1}^{i}\left(f_{i}\right)\right)$, where $\left(2^{\tau_{i}+\rho_{i-1}}\right)^{d} \varphi_{i-1}^{i}\left(f_{i-1}\right)$ and $\left.\left(2^{\tau_{i}+\rho_{i-1}}\right)^{d} \varphi_{i-1}^{i}\left(f_{i}\right)\right)$ are polynomials with integer coefficients. Furthermore, $\left(2^{\tau_{i}+\rho_{i-1}}\right)^{d} \varphi_{i-1}^{i}\left(f_{i-1}\right)$ is degree less than $d$ w.r.p.t $X_{1}^{i}$ and bit size less than $\tilde{\mathcal{O}}\left(2\left(\tau_{i}+\rho_{i-1}\right) d+\tau\right)=$ $\tilde{\mathcal{O}}\left(d \tau_{i}\right)$. Similarly, $\left(2^{\tau_{i}+\rho_{i-1}}\right)^{d} \varphi_{i-1}^{i}\left(f_{i}\right) \in \mathbb{Z}\left[\mathbf{x}_{i-2}, X_{i}^{i}, X_{i-1}^{i}\right]$ is degree less than $2 d$ w.r.p.t $X_{i-1}^{i}$ and bit size less than $\tilde{\mathcal{O}}\left(d \tau_{i}\right)$. According to Lemma 11, we compute $T_{2}^{i}\left(\mathbf{x}_{i-2}, X_{2}^{i}\right)$ in $\tilde{\mathcal{O}}_{B}\left(d(2 d+d)^{i}(2 d)^{i-1} d \tau_{i}\right)=\tilde{\mathcal{O}}_{B}\left(2^{i-1} 3^{i} d^{2 i+1} d^{i^{2}-2} \tau\right)=$ $\tilde{\mathcal{O}}_{B}\left(d^{i^{2}+2 i-1} \tau\right)$, and $\operatorname{deg}_{X_{2}^{i}}\left(T_{2}^{i}\right) \leq 3 d^{2}, \operatorname{deg}_{x_{j}}\left(T_{2}^{i}\right) \leq 2 d^{2}, j=1, \ldots, i-2$, bit size of $T_{2}^{i}$ is bounded by $\tilde{\mathcal{O}}\left(d^{2} \tau_{i}\right)=\tilde{\mathcal{O}}\left(d^{i^{2}} \tau\right), T_{2}^{i} \in \mathbb{Z}\left[\mathbf{x}_{i-2}, X_{2}^{i}\right]$.

Assume (d)(e) have been proved for $2,3, \ldots, l-1$.

For $l$, we compute $T_{l}^{i}$. Similarly to $l=2, T_{l}^{i}\left(X_{l}^{i}\right)=\operatorname{Res}_{X_{l-1}^{i}}$ $\left(\left(2^{\tau_{i-l+2}+\rho_{i-l+1}}\right)^{d} \varphi_{i-l+1}^{i}\left(f_{i-l+1}\right),\left(2^{\tau_{i-l+2}+\rho_{i-l+1}}\right)^{3^{l-2} d^{l-1}}\right.$ $\left.\varphi_{i-l+1}^{i}\left(T_{l-1}^{i}\right)\right)$, where $\left(2^{\tau_{i-l+2}+\rho_{i-l+1}}\right)^{d} \varphi_{i-l+1}^{i}\left(f_{i-l+1}\right)$ and $\left.\left(2^{\tau_{i-l+2}+\rho_{i-l+1}}\right)^{3^{l+2} d^{l-1}} \varphi_{i-l+1}^{i}\left(T_{l-1}^{i}\right)\right)$ are polynomials with integer coefficients. Furthermore, $\left(2^{\tau_{i-l+2}+\rho_{i-l+1}}\right)^{d} \varphi_{i-l+1}^{i}$

$\left(f_{i-l+1}\right)$ is degree less than $d$ w.r.p.t $X_{l-1}^{i}$, bit size less than $\tilde{\mathcal{O}}\left(2\left(\tau_{i-l+2}+\rho_{i-l+1}\right) d+\right.$ $\tau)=\tilde{\mathcal{O}}\left(d \tau_{i-l+2}\right)$. Similarly, $\left.\left(2^{\tau_{i-l+2}+\rho_{i-l+1}}\right)^{3^{l-2} d^{l-1}} \varphi_{i-l+1}^{i}\left(T_{l-1}^{i}\right)\right) \in \mathbb{Z}\left[\mathbf{x}_{i-l+2}, X_{l}^{i}, X_{l-1}^{i}\right]$ is degree less than $2 \cdot 3^{l-2} d^{l-1}$ w.r.p.t $X_{l-1}^{i}$ and bit size less than $\tilde{\mathcal{O}}\left(3^{l-2} d^{l-1} \tau_{i-l}+\right.$ $\left.d^{l-1} \tau_{i}\right)=\tilde{\mathcal{O}}\left(d^{l-1} \tau_{i}\right)$. According to Lemma 11 , we compute $T_{l}^{i}\left(X_{l}^{i}\right)$ in $\tilde{\mathcal{O}}_{B}\left(d\left(3^{l-2} d^{l-1}+\right.\right.$ $d)^{i-l+2}$ 
$\left.\left(3^{l-2} d^{l-1}\right)^{i-l+1} d^{l-1} \tau_{i}\right)=\tilde{\mathcal{O}}_{B}\left(d^{2(l-1) i--2 l^{2}+6 l-3} \tau_{i}\right)=\tilde{\mathcal{O}}_{B}$

$\left(d^{i^{2}+2(l-1) i-2 l^{2}+6 l-5} \tau\right)$, and $\operatorname{deg}_{X_{l}^{i}}\left(T_{l}^{i}\right) \leq 3^{l-1} d^{l}, \operatorname{deg}_{x_{j}}\left(T_{l}^{i}\right)$

$\leq 2^{l-1} d^{l}, j=1, \ldots, i-l$, bit size of $T_{l}^{i}$ is bounded by $\tilde{\mathcal{O}}\left(d^{l} \tau_{i}\right)=\tilde{\mathcal{O}}\left(d^{i^{2}+l-2} \tau\right)$, $T_{l}^{i} \in \mathbb{Z}\left[\mathbf{x}_{i-l}, X_{l}^{i}\right]$. So (d)(e) have been proved for $l$.

Furthermore, we compute $T_{i}^{i}$ in $\tilde{\mathcal{O}}_{B}\left(d^{i^{2}+4 i-5} \tau\right), \operatorname{deg}_{X_{i}^{i}}\left(T_{i}^{i}\right)$

$\leq 3^{i-1} d^{i}$, bit size of $T_{i}^{i}$ is bounded by $\tilde{\mathcal{O}}\left(d^{i^{2}+i-2} \tau\right)$. By Lemma 9 , we isolate $\left\{\eta_{i}\right\}$, the real roots of $T_{i}^{i}$ in $\tilde{\mathcal{O}}_{B}\left(\left(3^{i-1} d^{i}\right)^{3}\right.$

$\left.d^{i^{2}+2 i-3} \tau\right)=\tilde{\mathcal{O}}_{B}\left(d^{i^{2}+5 i-3} \tau\right)$, and the end points of the isolate intervals of them have bit size bounded by $\widetilde{\mathcal{O}}\left(3^{i-1} d^{i}\right.$

$\left.3 d^{i^{2}+i-2} \tau\right)=\tilde{\mathcal{O}}\left(d^{i^{2}+2 i-2} \tau\right)$. Then, we get back $\left\{\xi_{i}\right\}$ by $\xi_{i}=\frac{R_{i}}{r_{i-1}}\left(\eta_{i}-\xi_{i-1}\right)$, the bit size of $\xi_{2}$ are bounded by $\tilde{\mathcal{O}}\left(\tau_{i}+\rho_{i-1}+\mathcal{L}\left(\eta_{i}\right)\right)=\tilde{\mathcal{O}}\left(d^{i^{2}-2} \tau+d^{i^{2}-3} \tau+\right.$ $\left.d^{i^{2}+2 i-2} \tau\right)=\tilde{\mathcal{O}}\left(d^{i^{2}+2 i-2} \tau\right)$. So $\mathcal{L}\left(r_{i}\right) \leq \tilde{\mathcal{O}}\left(d^{i^{2}+2 i-2} \tau\right)$. According to Lemma 12, $\mathcal{L}\left(R_{i+1}\right) \leq \max \left\{\tilde{\mathcal{O}}\left(d^{i^{2}+2 i-1} \tau\right), \tilde{\mathcal{O}}_{B}\left(d^{i} \tau\right)\right\}=\tilde{\mathcal{O}}\left(d^{i^{2}+2 i-1} \tau\right)$. Obviously, this theorem have been proved.

Proof (of Thm. 6). In Algorithm 3, we need compute $R_{i}, T_{l}^{i}$ for $i=2, \ldots, n ; l=$ $2, \ldots, i$, and isolate the real roots of $T_{i}^{i}$ for $i=1, \ldots, n$. So the complexity of this algorithm is

$$
\begin{aligned}
& \sum_{i=1}^{n-1} \tilde{\mathcal{O}}_{B}\left(i d^{\frac{(i+2)^{2}}{2}} \tau\right) \\
& +\sum_{i=2}^{n} \sum_{l=2}^{i} \tilde{\mathcal{O}}_{B}\left(d^{i^{2}+(2 l-2) i-2 l^{2}+6 l-5} \tau\right) \\
& +\sum_{i=1}^{n} \tilde{\mathcal{O}}_{B}\left(d^{i^{2}+4 i-2} \tau\right)=\tilde{\mathcal{O}}_{B}\left(N^{n^{2}}\right) .
\end{aligned}
$$

